\title{
Simulation Analysis of Liquid Pipeline Flow under the Pre Pressurized Tank
}

\author{
Zhang Yuxiang ${ }^{1, a}$, Li Zhijia ${ }^{1, b}$, Zhang Xin ${ }^{1, c}$ \\ ${ }^{1}$ Xi'an Research Inst. Of Hi-Tech., Shanxi Xi'an, P. R. China 710025 \\ ayuxiangz@tom.com, bIzhjiaxx@163.com, '601549457@qq.com
}

Keywords: pressurized; flow; simulation; Fluent

\begin{abstract}
In some special applications of industrial production and aerospace field, the liquid pipeline flow under the pressurized tank is a problem which should be concerned in the design and application of the closed media delivery system [1]. Design Modeler and Meshing module in Workbench is used to establish the two-dimensional model of the liquid tank as well as pipeline and dividing the mesh. The boundary conditions are set and the flow simulation is carried out in Fluent. The results show that: due to internal pressure, external pressure, the interaction of gravity, inertia, and other factors, the entire flow process is divided into three stages, which are respectively the outflow of liquid and gas expansion stage, the liquid outflow and the backflow of gas stage and liquid phase opening. When the tank is connected with the outside world by the upper part of pipeline, liquid shows slow steady flow [2].
\end{abstract}

\section{Introduction}

A liquid flows outward from the tank through a pipeline is a common phenomenon in production and daily life. In some certain cases, due to the special requirements of the construction of tank, liquid physical properties and so on, the tank itself is a sealed tank. In order to ensure that the liquid can flow smoothly, advanced continuous ventilation or pre charge into a certain pressure gas is needed, so as to ensure that the liquid inside of the tank can outflow normally.

In the process of liquid rocket irrigation pump, first of all, it is needed to pre charge pressure for liquid tank, and the gas pipeline will be closed when the tank reaches a certain pressure. According to a certain rule to open the valve, liquid propellant flows out along the pipeline under gravity and the gas pressure within the tank, fills up the whole centrifugal pump and filling pipeline. The irrigation pump process is over when the correct full signal is output for the pump, then the next step of operation will be started [3].

According to the characteristics of the flow, Fluent, which is the large commercial simulation software, has been used in this paper. Based on the VOF model and the standard k- $\varepsilon$ turbulence model in Fluent, the situation simulation, can be completed, as well as studying the flow of the liquid line and impact of the export situation, and exploring the law of motion under the boundary conditions.

\section{Description of the flow equation [4]}

The VOF model is used to describe the liquid and gas two-phase flow in the tank. It is a turbulent flow in the tank due to both the gas pressure and liquid gravity, therefore the standard k- $\varepsilon$ turbulence model is chosen. A two dimensional model is established for the simulation, and the continuous equation and momentum equation is described as follows:

Continuous equation:

$$
\frac{\partial \rho}{\partial t}+u \frac{\partial(\rho u)}{\partial x}+v \frac{\partial(\rho v)}{\partial y}=0
$$

Momentum equation: 


$$
\left\{\begin{array}{l}
\rho \frac{d u}{d t}=\rho F_{b x}+\frac{\partial p_{x x}}{\partial x}+\frac{\partial p_{y x}}{\partial y} \\
\rho \frac{d v}{d t}=\rho F_{b y}+\frac{\partial p_{x y}}{\partial x}+\frac{\partial p_{y y}}{\partial y}
\end{array}\right.
$$

In the formula, $F_{b x}, F_{b y}$ is respectively the quality of force per unit mass on the direction of the 3 components and $p_{i j}(i, j=x, y)$ is the fluid stress tensor components.

\section{Entity modeling and boundary conditions [5]}

(1) To establish a two-dimensional model. In order to achieve the purpose of simulation and make the results more clear, according to the irrigation pump pipeline of a certain type of liquid missile propellant filling system, the model is reasonably simplified. Because the pipeline in the flow distribution is symmetrical, the symmetrical face for flow model is really representative for the study of liquid flow. Therefore, in this paper, the two-dimensional simulation model of symmetry plane is used to do the research, so that it can show flow process better, in the meantime the amount of calculation is also reduced. The two-dimensional model of the tank and pipeline is established by using the design modeler module of ANSYS Workbench. It is set the tank a square length of $2 \mathrm{~m}$, under the tank right part which is connected with the pipeline with height (diameter) of $0.4 \mathrm{~m}$ and long of 3m, so that the liquid can flow normally. A two-dimensional model is established as shown in Figure 1:

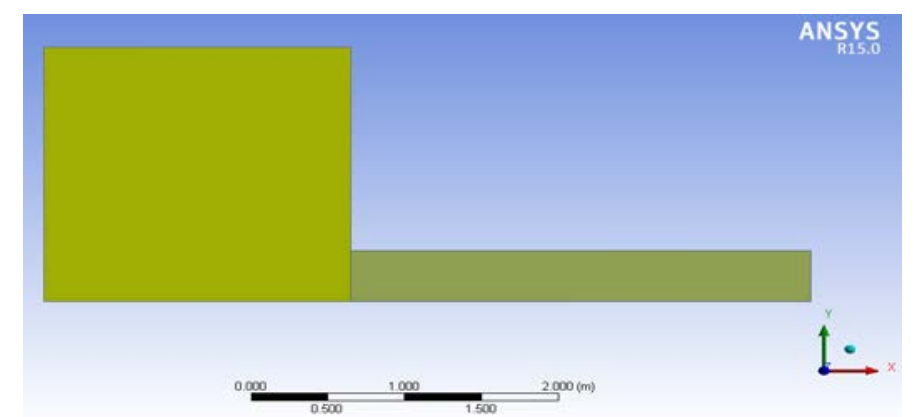

Fig. 1 The two dimensional model of pipeline flow under the pre pressurized tank

(2) The mesh generation. It is meshed by the meshing module of ANSYS Workbench. Due to the simple model rules, the structured mesh is chosen, and the mesh size is controlled to $20 \mathrm{~mm}$. The mesh is reached to 13000 and mesh effect is shown below in Figure 2.
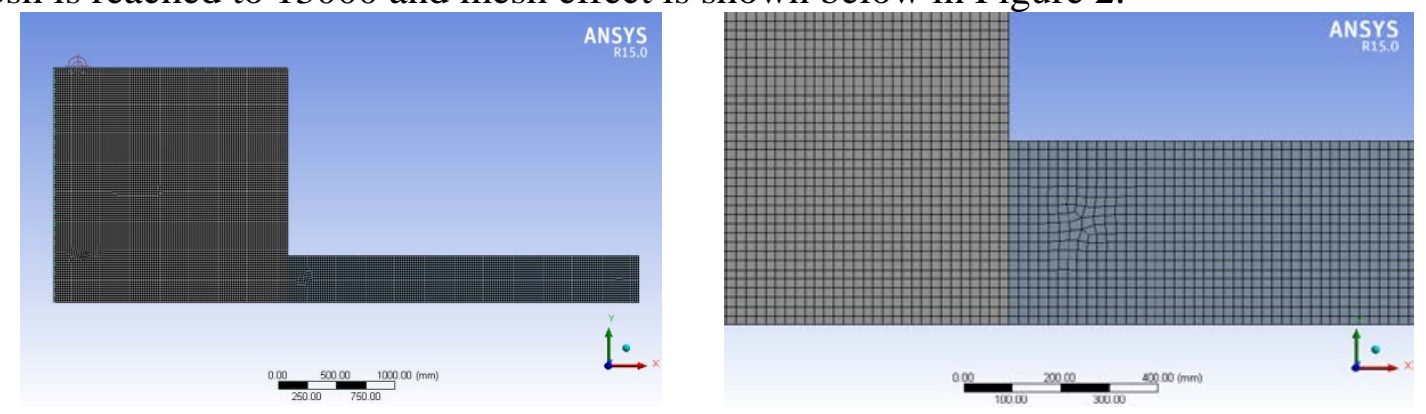

Fig. 2 The mesh generation

(3) The boundary conditions. The model is the pressurized flow model of tank, and there is no fluid inlet. The gas phase is the ideal air and the liquid phase is water. $\mathrm{In} 0 \mathrm{~m}$ to $1.5 \mathrm{~m}$ at the lower part of the tank is full of water, high upper $1.5 \mathrm{~m}-2 \mathrm{~m}$ with pressure air of $0.2 \mathrm{MPa}$. Initial conditions of the pipeline is connected with the external atmosphere, while the pipeline and tank are taken apart by a valve (due to the valve on the contents of no practical significance, it isn't reflected in the model). An outlet is set to the pressure-outlet whose value is a standard atmospheric pressure. The gravity is set to $9.8 \mathrm{~m} / \mathrm{s}^{2}$ along the negative direction of $\mathrm{Y}$ axis. 

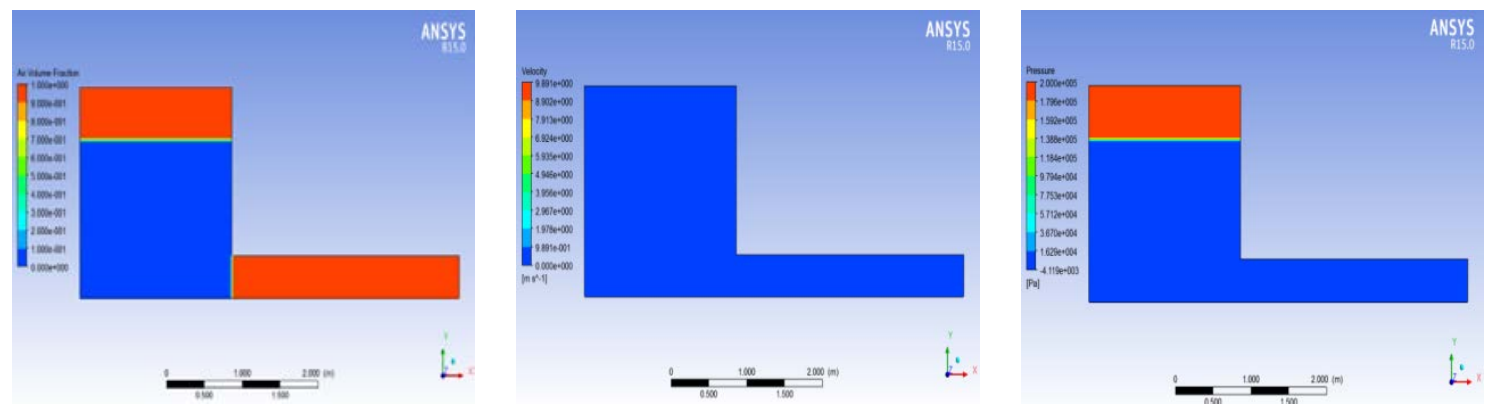

Fig. 3 Nephogram of gas-liquid phase distribution, velocity and pressure under the initial condition

\section{Simulation analysis of the flow field [6]}

According to the distribution of gas-liquid phase and gas reflux, the gas-liquid flow process is divided into three phases.

The first stage is 0s-0.9s. Under action of the gas pressure and its own gravity, liquid in tank overcome the atmospheric pressure outside the pipeline, flowing outward along the pipeline. In this stage, the liquid flows outward along the pipeline, and air is squeezed to the outside atmosphere. At this point, pressure of the tank at each position is greater than pressure in the pipeline, and gradually reducing from the inside to the outside, so there will be no gas reflux, but there are certain gas and liquid vortex in the pipeline. In addition, due to the outward flow of liquid, the gas and liquid pressure inside the tank are gradually reducing, and the flow velocity of the fluid in the pipeline has also been gradually reducing. But for the entire flow model, the velocity disturbance range is in increasing. Figure 4 is $0.2 \mathrm{~s}$ flow field of the gas-liquid distribution, velocity and pressure nephogram.
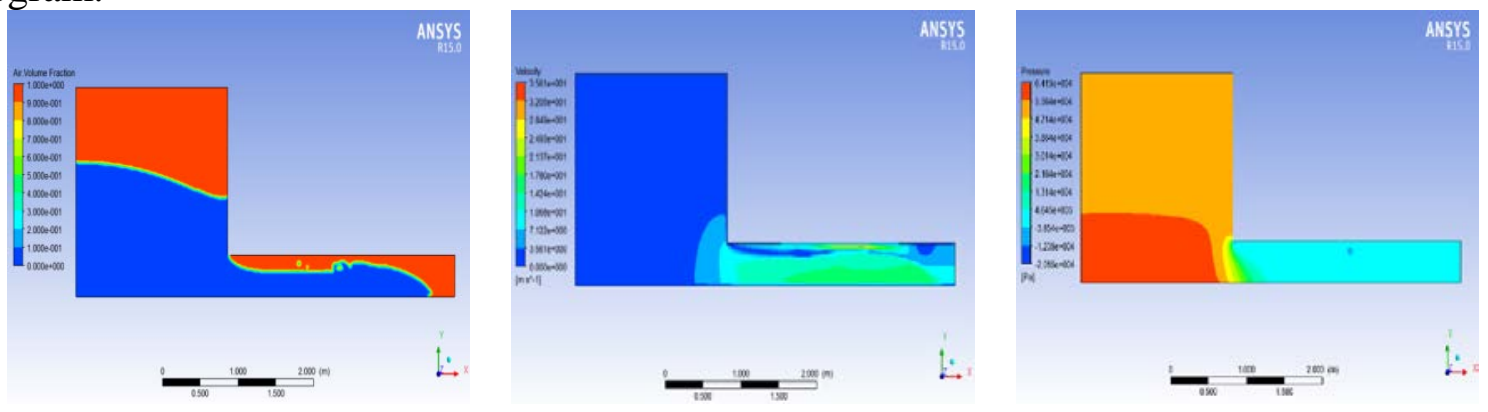

Fig. $4 \quad$ 0.2s flow field of the gas-liquid distribution, velocity and pressure nephogram

The second stage is $0.9 \mathrm{~s}-2.0 \mathrm{~s}$. When liquid flows outward, gas pressure in tank is gradually declining. When the flow is to a certain extent, the pressure in tank is less than the atmospheric pressure outside, then the gas return to the liquid tank along the upper part of the pipeline. After a period of time of development, gas is connected with the tank and gradually completely mixed. At this time, internal pressure of the pipeline is greater than internal pressure of the tank. Due to the effect of inertia and gravity, liquid still continue to flow outward, but the velocity decreases gradually. At some point, the tank forms a large negative pressure zone, so as to form an obvious gas reflux. Gas flows outward through the upper part of the pipeline and the liquid flows outward. Gas phase and liquid phase in the internal pipeline respectively flow along the different directions, with continuing strong gas reflux. The speed of the liquid in the tank disturbance is more obvious. Figure 5, Figure 6, is respectively, 0.9s and 1.4s flow field of the gas-liquid distribution, velocity and pressure nephogram. 

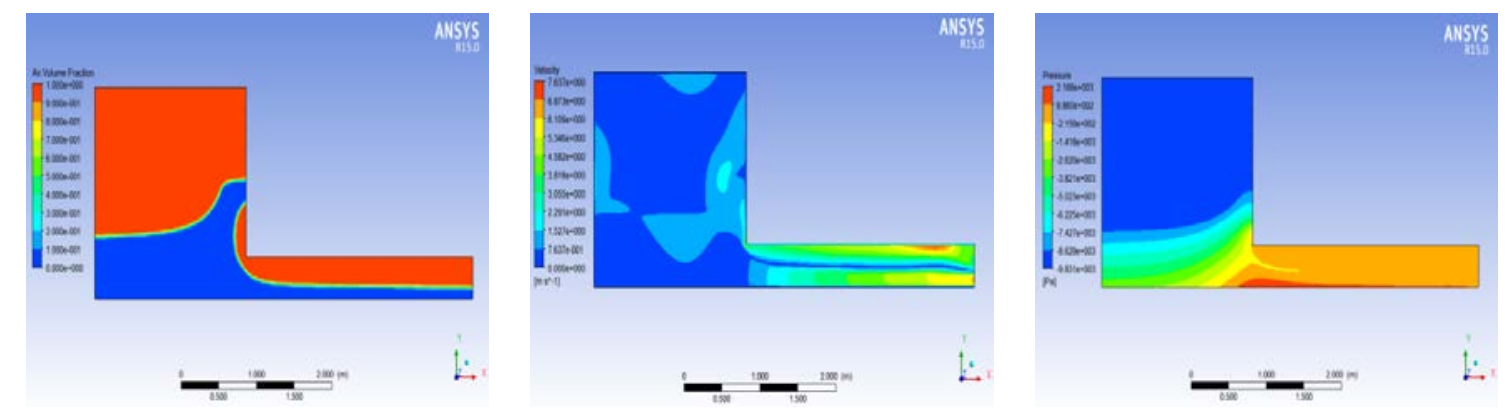

Fig. 5 0.9s flow field of the gas-liquid distribution, velocity and pressure nephogram
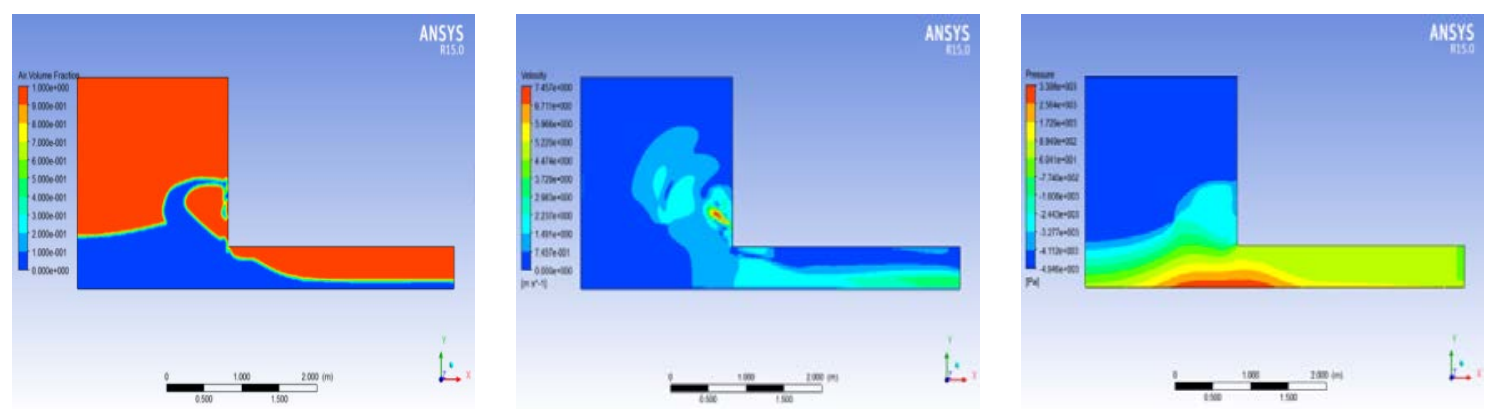

Fig. 6 1.4s flow field of the gas-liquid distribution, velocity and pressure nephogram

The third stage is 2.0s and later, at this point, the upper part of the pipeline and the gas in the liquid tank completely get through, and the model is close to when the liquid flow is in an open liquid tank. Mainly under the influence of its own gravity, liquid flows slowly, and fluid forms many irregular vortexes in the tank and pipeline. Velocity perturbation range of basic fills the tank. After a period of time (about 10s), the liquid flows out. As is shown in Figure 7, figure 8 and figure 9 about 2s, 5s and 10s, the flow field of the gas-liquid distribution, velocity and pressure nephogram.
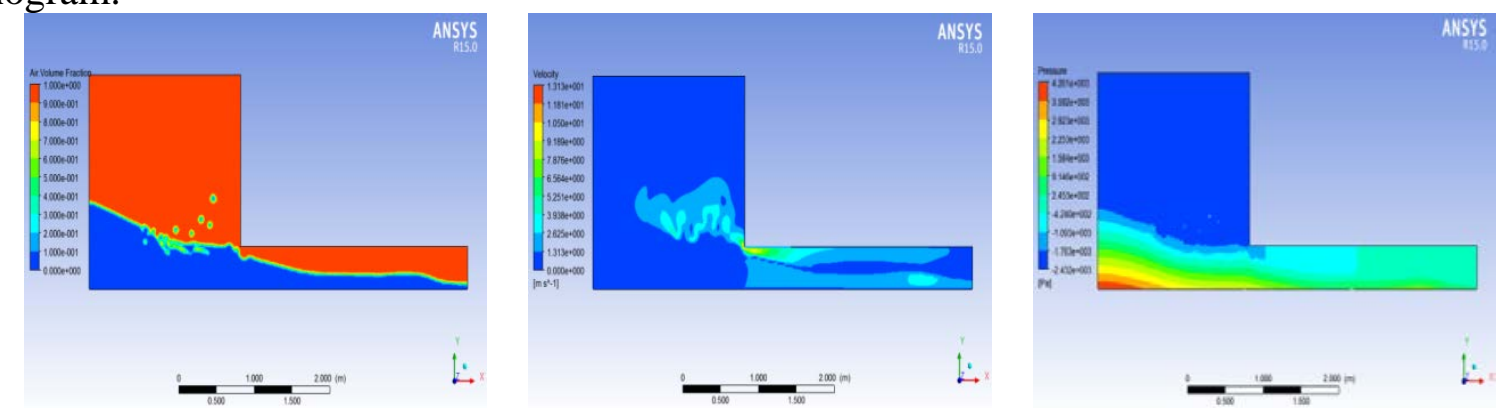

Fig. $7 \quad$ 2s flow field of the gas-liquid distribution, velocity and pressure nephogram
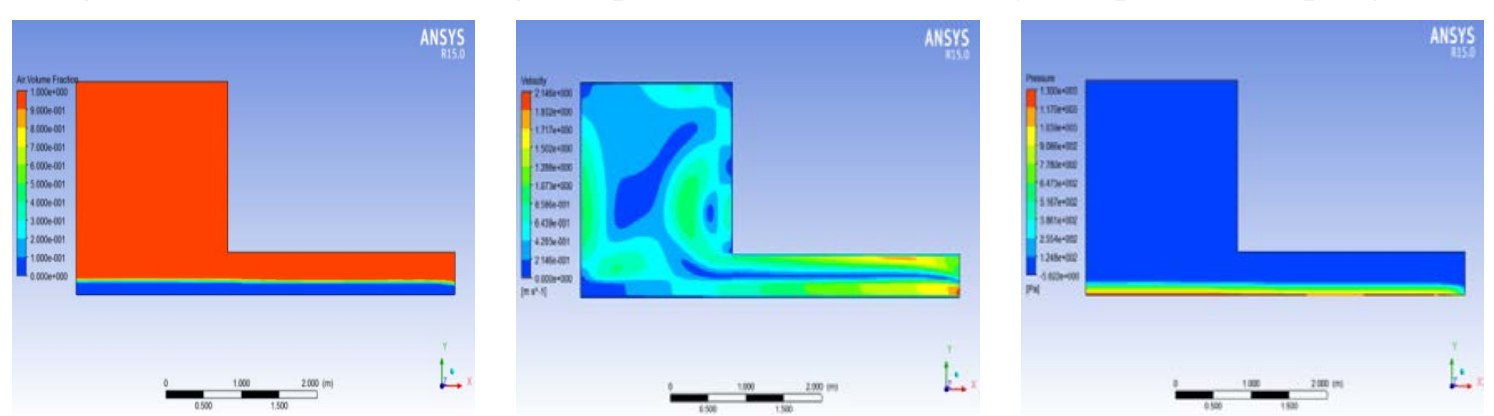

Fig. 8 5s flow field of the gas-liquid distribution, velocity and pressure nephogram 

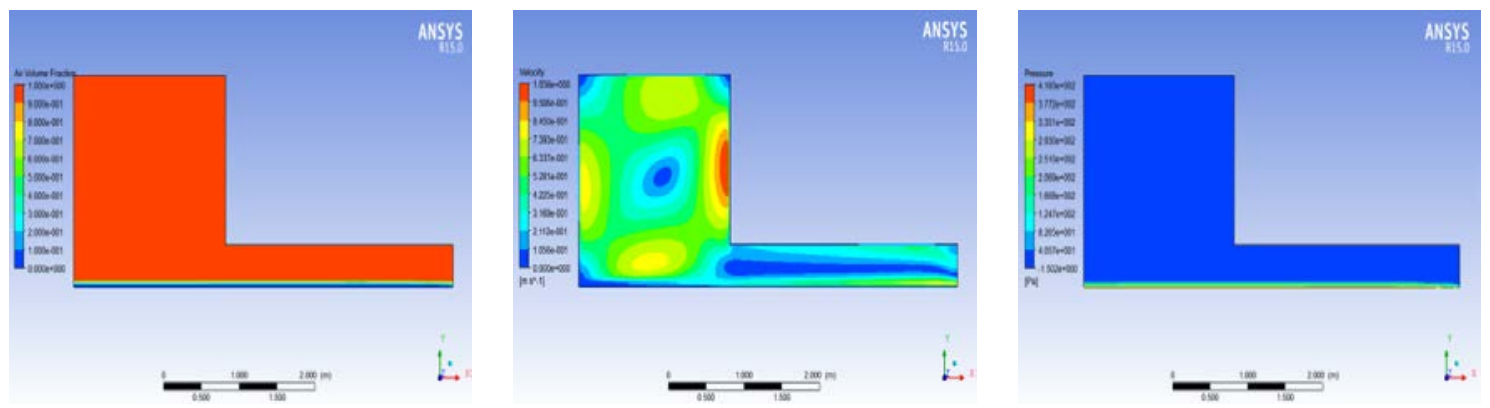

Fig. 9 10s flow field of the gas-liquid distribution, velocity and pressure nephogram

Monitor the velocity of the following three points: $0.1,0.2,0.3 \mathrm{~m}$ height on the tank outlet position in the whole flow process, and the velocity curve is as shown in Figure 10. It shows that, fluid in the pipeline flows to external at high speed at first. When it goes to the second stage, gas flows into the pipe in the upper part, while the liquid continues to flow outward in the lower part, and the velocity decreases gradually. When it arrives at the third stage, the velocity has a smaller increase, but flow is always in the same direction and gradually tends to 0 .

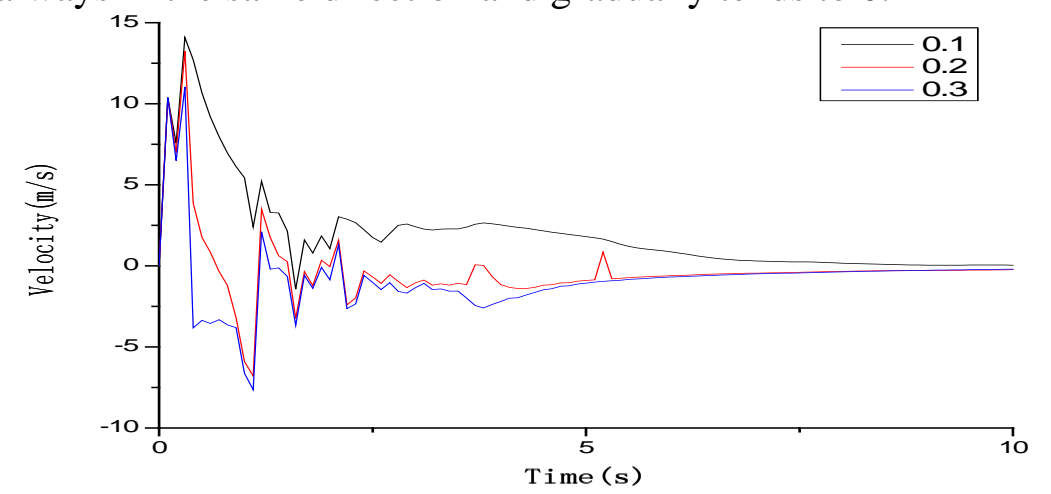

Fig.10 Velocity distribution of outlet position under different height

\section{Conclusions and outlook [7]}

To sum up, the flow model of the pipeline under the condition of the pre pressurized is simulated and completed and the conclusion is as follows:

(1) While the gas with enough pressure has been filled in the liquid tank, the flow patterns can be divided into three stages: liquid outflow and gas expansion on the influence of the gas pressure and gravity, the liquid outflow and the gas backflow on the influence of inertia and internal and external pressure as well as the gas backflow and the open flow of liquid tank connected with the outside world.

(2) At the beginning of the flow, the tank pressure gradually decreases, but has been higher than the pressure in the pipeline. With the flow going down, a negative pressure zone is formed in the tank, leading to flow of pipe gas.

(3) When the gas has been returned to a certain degree, the reflux gas in the upper part of pipeline is connected with the gas in the tank, and the air outside can be quickly poured into the liquid tank, which can balance air pressure inside and outside.

(4) The flow velocity of the fluid in the outlet of the pipeline is divided into three stages according to the flow state, and it presents different trends at different stages.

In the future part of the study, it is also needed to consider the different gas pressure in the liquid tank, the change of the pipe diameter, so as to have a more comprehensive understanding of the gas flow in advance.

\section{Acknowledgements}

This work was financially supported by The National Natural Science Fund (51275517). 


\section{Reference}

[1] ZHOU Zhi-jin, YANG Ning and WANG Zhao. Analysis on Shock Wave Speed of Water Hammer of Lifting Pipes for Deep-Sea Mining [J].China Ocean Eng. , 27(2), 2013: 205-214

[2] Park S H, Kang Y, Kim S D. Wavelet transform analysis of pressure fluctuation signals in a pressurized bubble column [J]. Chem. Eng. Sci. ,2001,56:6259-6265

[3] WU Min-sheng, DUAN Wen-guang. The application of computational fluid dynamics (CFD) in the field of the fluid machinery [J]. Equipment Manufacturing Technology,2008(12):36-37

[4] John Kanute. Self-priming Pump centrifugal pumps a primer [J]. World pump, 9,2004: 30-32

[5] Gong Yu, Gui Lin, Xu Yesong. Numerical simulation of ball valve resistance coefficient and flow field analysis [J]. Water Power Technology, (9), 2010:19-24

[6] Gao Z K, Jin N D, Flow pattern identification and nonlinear dynamics of gas-liquid two phase flow in complex networks [J]. Phys. Rev. E, 2009,79:066303-06313

[7] Rouhani S Z, Sohal M S. Two-phase flow patterns: a review of research results [J]. Prog. Nucl. Energy, 1983,11:219-259 